\title{
Munchausen's syndrome presenting as hemoptysis in a 12-year-old girl
}

\author{
Candice L Bjornson MD FRCPC, Valerie G Kirk MD FRCPC \\ Department of Pediatrics, University of Calgary, Calgary, Alberta
}

\section{Bjornson, VG Kirk. Munchausen's syndrome pre- senting as hemoptysis in a 12-year-old girl. Can Respir J 2001;8(6):439-442.}

The case of Munchausen's syndrome presenting as hemoptysis in a 12-year-old girl is presented. The features of Munchausen's syndrome are reviewed. Munchausen's syndrome should be included in the differential diagnosis of hemoptysis in a child, especially when accompanied by a dramatic presentation, changing symptoms and negative diagnostic investigations.

Key Words: Children; Factitious disorder; Hemoptysis; Munchausen's syndrome

\section{Syndrome de Münchhausen se manifestant sous forme d'hémoptysie chez une fillette de 12 ans}

RÉSUMÉ : Voici un cas de syndrome de Münchhausen se manifestant sous forme d'hémoptysie chez une fillette de 12 ans. Le présent article passe en revue les principaux éléments du syndrome. Celui-ci devrait être envisagé dans le diagnostic différentiel de l'hémoptysie chez les enfants, surtout lorsqu'on est devant une présentation dramatique, des symptômes changeants et des examens diagnostiques négatifs.

\begin{abstract}
A lthough Munchausen's syndrome and factitious disorder are listed as causes of hemoptysis in pediatric pulmonary medicine textbooks $(1,2)$, there are no published reports of Munchausen's syndrome presenting as hemoptysis in children. According to the Diagnostic and Statistical Manual of Mental Disorders (3), factitious disorder is the intentional production of physical or psychological signs or symptoms. The motivation for the behaviour is to assume the sick role in the absence of external incentives (eg, economic gain, avoiding legal responsibility or improving
\end{abstract}

physical well-being) for the behaviour (3). Munchausen's syndrome has been described as a chronic and severe subgroup of the factitious disorders, with predominantly physical signs and symptoms (3). However, within the field, there is inconsistency in the nomenclature, and the term Munchausen's syndrome is often used as a synonym for factitious disorder (4). In the present case report, we describe the case of a 12-year-old girl who presented to the pediatric respirology service with hemoptysis and was subsequently diagnosed with Munchausen's syndrome. 


\section{CASE PRESENTATION}

A 12-year-old girl presented to the Emergency Department of the Alberta Children's Hospital (Calgary, Alberta) with a two-month history of hematemesis and epigastric pain. She was admitted to hospital after consultation by the gastroenterology service. Extensive investigations were normal, including an upper endoscopy. On discharge from hospital, there was no specific follow-up appointment arranged at the Alberta Children's Hospital gastroenterology clinic, and the patient was referred back to her family physician for ongoing care. She then was seen in consultation by a gastroenterologist at the Rockyview General Hospital (Calgary, Alberta) for similar complaints four weeks after her initial presentation. Repeat investigations were normal, including a second upper endoscopy. The patient's parents then contacted the gastroenterologist at the Alberta Children's Hospital because she had developed a persistent cough that was productive of moderate amounts of fresh blood. The gastroenterologist made a direct referral to the pediatric respirology service. She arrived at the clinic accompanied by both parents, who were extremely concerned after witnessing several episodes of frank hemoptysis.

On detailed history, associated symptoms included undocumented fevers, unwitnessed 'blackouts', abdominal pain and blood-streaked diarrhea. She had no history of chest trauma or pain, previous hemoptysis or cough, infectious contacts, recent travel or medication use. She did report the onset of her menarche and two episodes of epistaxis coinciding with the first episode of hemoptysis. Her medical history was unremarkable, and her family history was negative for bleeding diatheses, pulmonary disease or gastrointestinal disease. Socially, the patient was described as a model student, but had missed two months of school due to her symptoms. Her parents reported financial difficulties because of missed time from work to care for their child.

Physical examination revealed a cheerful girl in no distress. Her vital signs were normal, and her weight and height were appropriate for her age. A head and neck examination included inspection of the nasal and oral mucosa. The findings were limited to an ulcerated lesion in the left nostril that was not actively bleeding, and the oropharyngeal mucosa appeared normal with no obvious bleeding or telangectasia. A chest examination was completely normal except for a dry, intermittent cough, which the patient was able to suppress on request. An abdominal examination revealed mild, diffuse tenderness, but was otherwise normal. The remainder of the examination was normal; specifically, there were no petechiae, ecchymoses, telangiectasia or finger clubbing. While left alone with her parents in the examination room, the patient expectorated approximately $30 \mathrm{~mL}$ of bloody fluid, which was left in a cup for the physician to view.

She was readmitted to hospital. The initial investigations included a normal complete blood count and differential, and a normal urinalysis. Full pulmonary function tests (spirometry, lung volume measurements and diffusion capacity) were completely normal. Flexible bronchoscopy was performed on the day of presentation, and showed normal anatomy and no active pulmonary bleeding. Apart from the previously described lesion in the left nostril, the nasal mucosa was normal. The oral cavity was examined thoroughly. When her lower lip was retracted fully, numerous fresh-appearing lesions that appeared consistent with a biting injury were found deep on the innermost aspect of the lower lip buccal mucosa. Bronchoalveolar lavage fluid was sent for bacterial culture, stain and culture for acid-fast bacilli, and stain for hemosiderin-laden macrophages. All results were negative.

On the ward that evening, the patient continued to have episodes of hemoptysis, after which she would summon the nursing staff by call bell to view the bloody fluid. Her parents were informed of our suspicions of factitious hemoptysis based on the unusual presentation, negative investigations and the discovery of the mouth lesions. A referral to the psychology service was made with the full support of the patient's parents. The psychologist's assessment revealed further relevant history. During a joint interview with her parents, the patient described herself as a "good student" with "lots of friends", and she described no major stresses at home or school. However, when interviewed alone, the patient admitted to having difficulties at school with other children "bullying" her. She also described significant conflicts with her mother, a claim that was subsequently confirmed by her parents. It was also discovered that the patient had made a disclosure of physical conflicts with her mother to her school guidance counsellor one month before her symptoms began. The counsellor immediately reported the situation to the Department of Child Welfare. When interviewed by the department's social worker, the patient stated that there were no difficulties at home, and after a complete investigation, the file was closed.

As part of the psychology assessment, the patient completed the Child Depression Scale and the Child Manifest Anxiety Scale. Her scores did not fall within the clinically significant range on either scale. During this assessment, the psychologist discussed with the patient the need for her to return to school immediately. Although her parents were also strongly supportive of her return to school, the patient appeared very upset and explained that she wanted to stay in hospital where "the nurses make me feel good".

On the second evening of admission, a nurse witnessed the patient forcibly driving her finger into her nostril and placing the blood from her finger into her mouth while holding the call bell in her other hand. The patient became extremely angry when confronted by the nurse, calling the nurse a "liar". The psychiatry service was consulted the following day. The information obtained by the psychiatrist corroborated the history in the psychology assessment. In addition, the psychiatric history revealed no history of psychiatric illness in the patient nor in the family. During the interview, the patient denied any history of physical or sexual abuse, although the report described a "lack of family cohesion" and again described a history of conflicts at home. The patient did not 
openly admit to self-induced behaviour causing her illness, and said that she was upset with the nursing staff for suggesting that she was "faking" her symptoms. The mental status examination was normal, and there was no evidence of depression, anxiety, psychosis or obsessive-compulsive behaviours. After reviewing the case, the diagnosis of Munchausen's syndrome was made by the psychiatry service. Care was transferred to the Department of Psychiatry, where the patient is currently receiving treatment.

\section{DISCUSSION}

Information regarding the prevalence of factitious disorder and Munchausen's syndrome is limited, probably because the disease is often unrecognized and difficult to diagnose (3). In large, general hospitals, factitious disorder is diagnosed in approximately $1 \%$ of all patients assessed by mental health professionals; the subgroup 'with predominantly physical signs and symptoms' predominates (3). Factitious disorders are more common in female individuals. However, male individuals appear more likely than female individuals to be diagnosed with Munchausen's syndrome if the strict diagnostic features describing Munchausen's syndrome as the chronic and severe form of factitious disorder are used (3). Munchausen's syndrome occurs predominantly in adult men in their late 30 s, but the syndrome has been described in younger adolescents who are often female (5).

We report the first case of Munchausen's syndrome presenting with hemoptysis in a child. In the pediatric age group, there is a case report in the literature of Munchausen's by proxy presenting in infant twins with apparent hemoptysis (6). However, a search of the literature revealed no previously described cases of Munchausen's syndrome presenting with hemoptysis in the pediatric population. Previously published cases have described adults who have presented with a variety of unusual histories and simulations of serious pulmonary diseases (7-16). The presentation of symptoms and complaints can be impressive, and can be a diagnostic challenge to each newly involved and unsuspecting physician. On review of the published case reports, we found separate reports from two institutions describing an eerily similar case of Munchausen's syndrome presenting with hemoptysis in an adult male $(15,16)$. Observant readers questioned a possible link in these two cases $(17,18)$, and the subject in question was eventually determined to be the same person (19).

Factitious disorders are "characterized by physical or psychological symptoms that are intentionally produced or feigned in order to assume the sick role" (3). The three diagnostic criteria for factitious disorder are the intentional production of physical or psychological signs or symptoms (criterion A); the motivation for the behaviour is to assume the sick role (criterion $\mathrm{B}$ ); and external incentives for the behaviour are absent (criterion C) (3). As described in the Diagnostic and Statistical Manual of Mental Disorders (3), Munchausen's syndrome is the most severe and chronic subgroup of factitious disorders, but the terms are often used interchangeably (4). The pertinent diagnostic features of Munchausen's syndrome are simulation of severe illness requiring hospitalization, spurious signs of disease that may be due to self-injury, multiple hospitalizations at geographically widely separated institutions, pathological lying, aggressive or evasive behaviour, and self-discharge from hospital, usually against medical advice (20). Evidence for the diagnosis of Munchausen's syndrome in our patient is as follows: she presented dramatically with a serious complaint caused by self-injury, showed evidence of lying to her parents and medical personnel, gained admission and had invasive procedures performed at two institutions, and demonstrated aggressive behaviour when confronted by nursing personnel. Clearly, some features of Munchausen's syndrome would be difficult for a child to manifest. For example, children cannot travel great distances to separate hospitals and are unlikely to discharge themselves from medical care due to dependence on their parents.

Munchausen's syndrome should be included in the differential diagnosis of hemoptysis in a child, especially when accompanied by a dramatic presentation, changing symptoms and negative medical investigations. When evaluating a patient with hemoptysis, we recommend that clinicians modify the physical examination of the oropharynx and nasal cavity, and perform an exhaustive inspection to rule out any self-inflicted sources of bleeding that may be hidden in areas that are difficult to see. Psychiatric consultation is essential in cases of Munchausen's syndrome. Treatment is often attempted using psychotherapy. Unfortunately, treatment has been noted to be very difficult, and there are many patients for whom a cure remains elusive (4). However, with early recognition, patients may be protected from unnecessary invasive investigations and hospitalizations, and prompt referrals for appropriate counselling and treatment can be made.

\section{REFERENCES}

1. Brown M, Morgan W. Clinical assessment and diagnostic approach to common problems. In: Taussig L, Landau L, eds. Pediatric Respiratory Medicine. St Louis: Mosby, 1999:136-52.

2. Rosenstein B. Hemoptysis. In: Hillman B, ed. Pediatric Respiratory Disease. Diagnosis and Treatment. Philadelphia: WB Saunders, 1993:533-43.

3. Hales R, Cloninger C, Borus J, et al. Factitious disorders. In: Diagnostic and Statistical Manual of Mental Disorders, 4th edn. Text Revision. Washington, DC: American Psychiatric Association, 2000:513-7.

4. Nadelson T. The false patient: Chronic factitious disease, Munchausen syndrome, and malingering. In: Freeman J, ed. Psychiatry. Philadelphia: JB Lippincott Company, 1994:1-11.

5. Raymond C. Munchausen's may occur in younger persons. JAMA 1987;257:3332. (Lett)

6. Lee D. Munchausen syndrome by proxy in twins. Arch Dis Child 1979;54:646-7.

7. Roethe R, Fuller P, Byrd R, Stanford W, Fisk D. Munchausen syndrome with pulmonary manifestations. Chest 1981;79:487-8.

8. Mitchell D, Doyle P, Spiro S. Munchausen's syndrome with multiple pulmonary manifestations. J R Soc Med 1985;78:681-2.

9. Carroll D. Obscure causes of hemoptysis. Md State Med J 1975;24:87-8.

10. Seguel M, Arrese M, Perez C, Alverez M, Labarca R, Veloso M. Munchausen syndrome: A report of 6 patients. Rev Med Chil 1990;118:1090-7. 


\section{Bjornson and Kirk}

11. Feinsilver S, Raffin T, Kornei M, Sullivan S, Smith M. Factitious hemoptysis: The case of the red towel. Arch Intern Med 1983;143:567-8.

12. Rusakow L, Gershan W, Bulto M, Szajnberg N. Munchausen's syndrome presenting as cystic fibrosis with hemoptysis. Pediatr Pulmonol 1993;16:326-9.

13. Okimoto N, Soejima R. Factitious hemoptysis and anemia. Chest 1994;105:1629. (Lett)

14. Baktari J, Tashkin D, Small G. Factitious hemoptysis: Adding to the differential diagnosis. Chest 1994;105:943-5.
15. Duffy T. The Red Baron. N Engl J Med 1992;327:408-11.

16. Flynn J. Munchausen syndrome. N Y State J Med 1992;92:301-5.

17. Imperato P. Kidney-related Munchausen's syndrome and the Red Baron. N Engl J Med 1993;328:60. (Lett)

18. Weltman A. Kidney-related Munchausen's syndrome and the Red Baron. N Engl J Med 1993;328:61. (Lett)

19. Duffy T. Kidney-related Munchausen's syndrome and the Red Baron. N Engl J Med 1993;328:60-1. (Lett)

20. Folks D, Freeman A. Munchausen's syndrome and other factitious illness. Psychiatr Clin North Am 1985;8:263-78. 


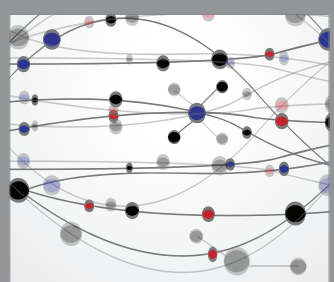

The Scientific World Journal
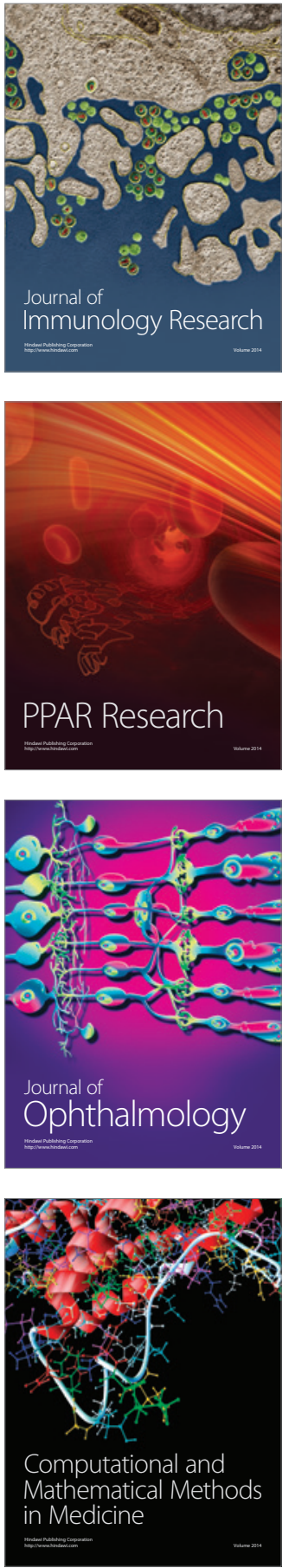

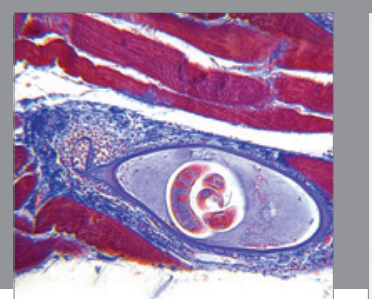

Gastroenterology Research and Practice

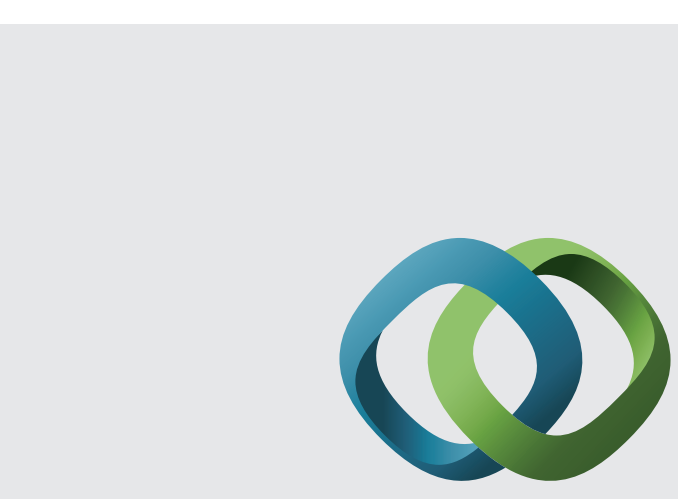

\section{Hindawi}

Submit your manuscripts at

http://www.hindawi.com
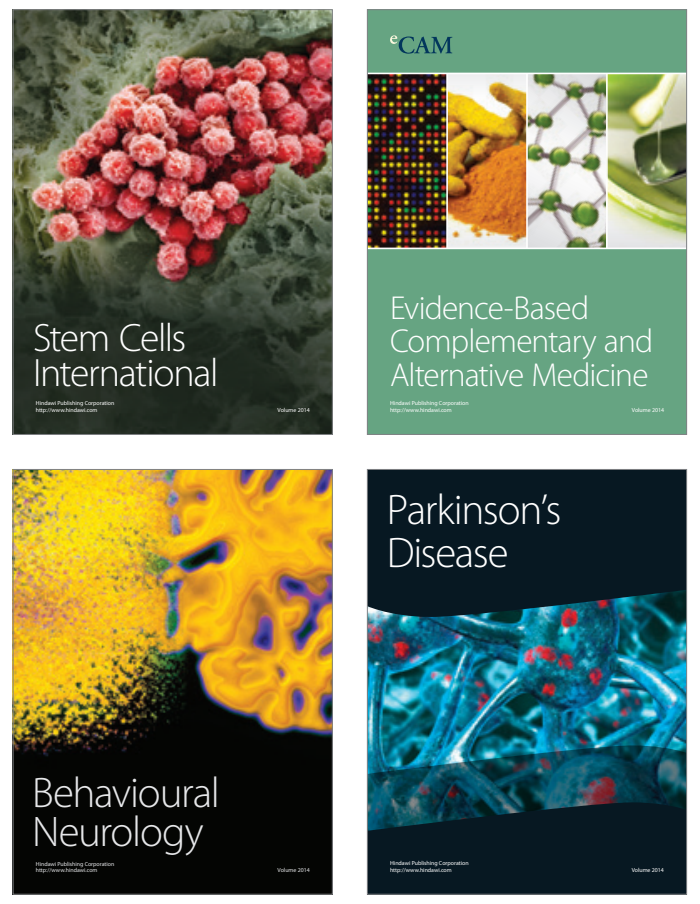
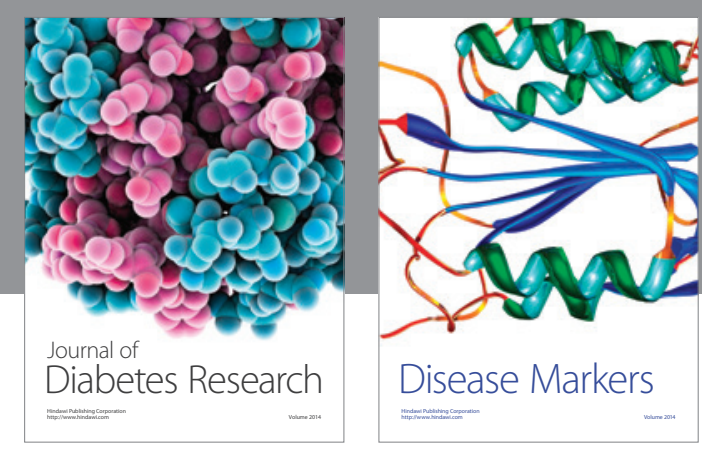

Disease Markers
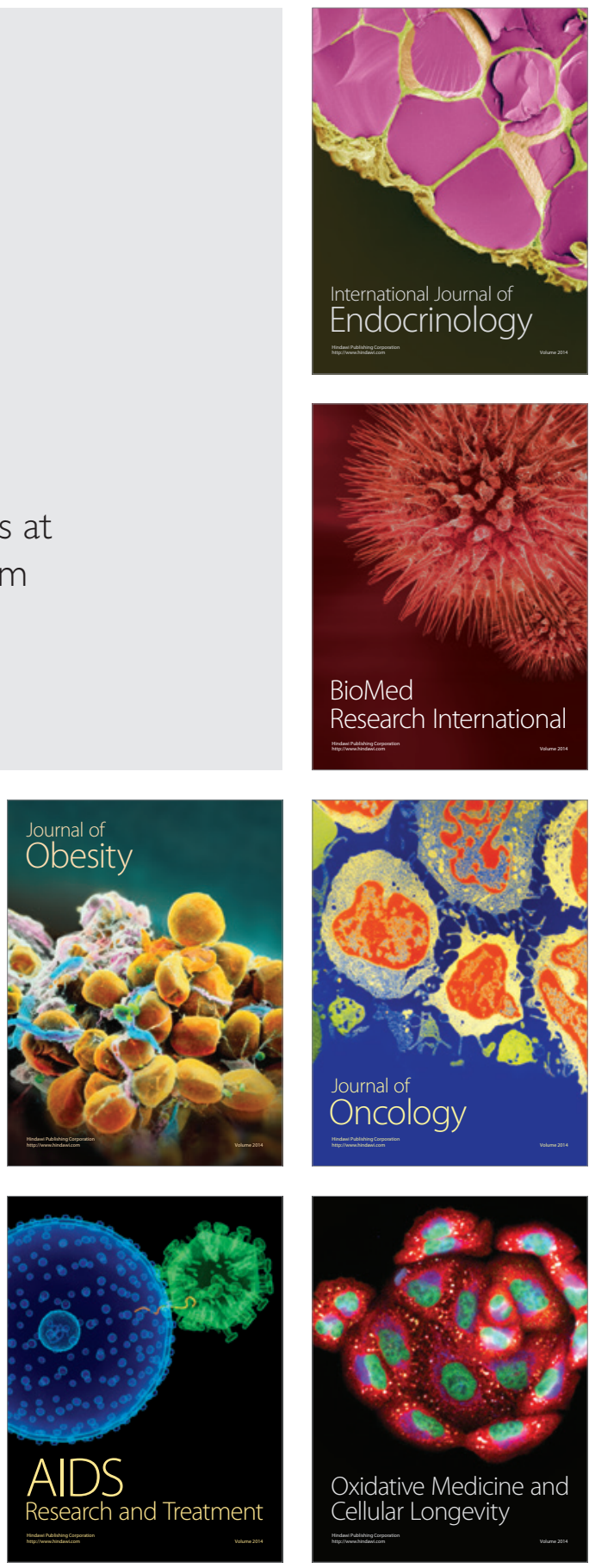